\title{
Evidence of Early Stage Precursors of Polymer Crystals by Dielectric Spectroscopy
}

\author{
M. Soccio, ${ }^{1}$ A. Nogales, ${ }^{2}$ N. Lotti, ${ }^{1}$ A. Munari, ${ }^{1}$ and T. A. Ezquerra ${ }^{2}$ \\ ${ }^{1}$ Dipartimento di Chimica Applicata e Scienza dei Materiali, Universitá di Bologna, Via Risorgimento 2, 40136 Bologna, Italy \\ ${ }^{2}$ Instituto de Estructura de la Materia, CSIC, Serrano 121, Madrid 28006, Spain
}

(Received 30 August 2006; published 17 January 2007)

\begin{abstract}
Dielectric spectra of the polyester poly(propylene succinate) were measured upon crystallization. For this model aliphatic polyester the $\alpha$ and $\beta$ relaxations appear simultaneously and are well resolved in the experimental frequency window. During isothermal crystallization, this fact allows one to use the $\beta$ relaxation to characterize the crystalline structural development while the $\alpha$ relaxation provides information about the evolution of the amorphous phase dynamics. In this way structure development and dynamics evolution can be characterized by a single experiment during the crystallization process. The unambiguous analysis of the dielectric loss clearly supports the existence of precursors of crystallization in the induction period.
\end{abstract}

DOI: 10.1103/PhysRevLett.98.037801

PACS numbers: 61.41.+e, 64.60.Qb, 77.22.Gm

Recent experimental [1-4], theoretical [4-6], and simulation [7] works have given considerable effort to show the possibility that the formation of a crystalline phase in polymers may be preceded by precursor phases with polymer backbone rigidity different to that of the initial amorphous phase. The experimental evidence for this proposal originates from time-resolved $\mathrm{x}$-ray experiments showing that an excess of scattering at small angles precedes the appearance of Bragg peaks at wide angles [1,2,8]. However, significant criticism of the precursor hypothesis has also been presented by several experimental $[9,10]$ and simulation [11] papers which favor the traditional nucleation and growth scenario. Here, we use dielectric spectroscopy [12] as a relaxation technique capable to probe polymer chain dynamics in the induction period before crystallization. Our novel approach includes the specific synthesis of a model aliphatic polyester, poly(propylene succinate) in which the $\alpha$ relaxation, related to the segmental dynamics [12] and very sensitive to ordering processes [13], appears in the measured frequency range together, but still well resolved, with the $\beta$ relaxation associated with the local dynamics and linearly related to the amount of the crystalline phase [14]. This fact allows one to characterize simultaneously structure development and dynamics by a single experiment. Our experiments reveal an increase of the polymer backbone correlation dynamics in the induction period thus favoring the precursor hypothesis.

Poly(propylene succinate) (PPS) [see scheme in Fig. 1(a)] was synthesized as described elsewhere [15]. The polymer was obtained with a molecular weight of $M_{n}=36.000 \mathrm{~g} / \mathrm{mol}$, a calorimetric glass transition temperature of $T_{g}=-27^{\circ} \mathrm{C}$, and a melting temperature of $T_{m}=44^{\circ} \mathrm{C}$. Dielectric spectroscopy measures the complex dielectric permittivity $\varepsilon^{*}=\varepsilon^{\prime}-\varepsilon^{\prime \prime}$ as a function of frequency, where $\varepsilon^{\prime}$ is the dielectric constant and $\varepsilon^{\prime \prime}$ is the dielectric loss [12]. Specimens for dielectric spectroscopy were pressed between two circular metallic elec- trodes of $2 \mathrm{~cm}$ diameter at $100^{\circ} \mathrm{C}$. Kapton spacers $0.07 \mathrm{~mm}$ thick were used to avoid the short circuit of the two metallic electrodes. The sample was then quenched at room temperature and immediately transferred to the dielectric cell. Dielectric loss measurements $\varepsilon^{\prime \prime}=\operatorname{Im}\left(\varepsilon^{*}\right)$ were performed over a broad frequency window, $10^{-1}<$ $F(\mathrm{~Hz})<10^{7}$, by means of a Novocontrol system integrating a dielectric interface (ALPHA) and a temperature control by nitrogen jet (QUATRO) with a temperature error, during every single sweep in frequency, of $0.1 \mathrm{~K}$. In order to follow the crystallization process, the sample was held at $25^{\circ} \mathrm{C}$. After a controlled period of time the sample was rapidly cooled down to $-25^{\circ} \mathrm{C}$ at $5{ }^{\circ} \mathrm{C} / \mathrm{min}$ to perform a frequency swept ( $2 \mathrm{~min}$ ) and immediately heated up to $25^{\circ} \mathrm{C}$ at $5{ }^{\circ} \mathrm{C} / \mathrm{min}$ to wait for another time period. Several cycles were first performed in order to remove internal stresses in the sample before starting to count the time. The crystallinity for the initial sample and of that after the isothermal crystallization experiment was estimated by means of wide angle x-ray scattering (WAXS) [Seifert XRD $3000 \theta / \theta$ diffractometer using Ni-filtered $\mathrm{Cu}$ $K \alpha$ radiation $(\lambda=0.154 \mathrm{~nm})]$. Figure 1 (a) shows that PPS at $T>T_{g}$ exhibits the two main relaxation processes characteristic of most polymers, $\alpha$ and $\beta$, in order of increasing frequency. Figures 1(a) and 1(b) present the $\varepsilon^{\prime \prime}$ data at $-25^{\circ} \mathrm{C}$ as a function of frequency $F=\omega /(2 \pi), \omega$ being the angular frequency, taken after different periods of holding time at $25^{\circ} \mathrm{C}$. The initial amorphous state is characterized by an intense relaxation, $\alpha$, associated with the segmental dynamics [12], and a less intense relaxation, $\beta$, related to the local chain dynamics $[12,14]$. As time increases there are two clear regimes: an initial one [Fig. 1(a)] where the $\alpha$ relaxation exhibits a decrease of its intensity with crystallization time while the $\beta$ relaxation remains unaffected, and a second regime [Fig. 1(b)] where both $\alpha$ and $\beta$ relaxations decrease with time and additionally the $\alpha$ relaxation exhibits a shift towards lower frequencies. The shape analysis of the dielectric curves can be 

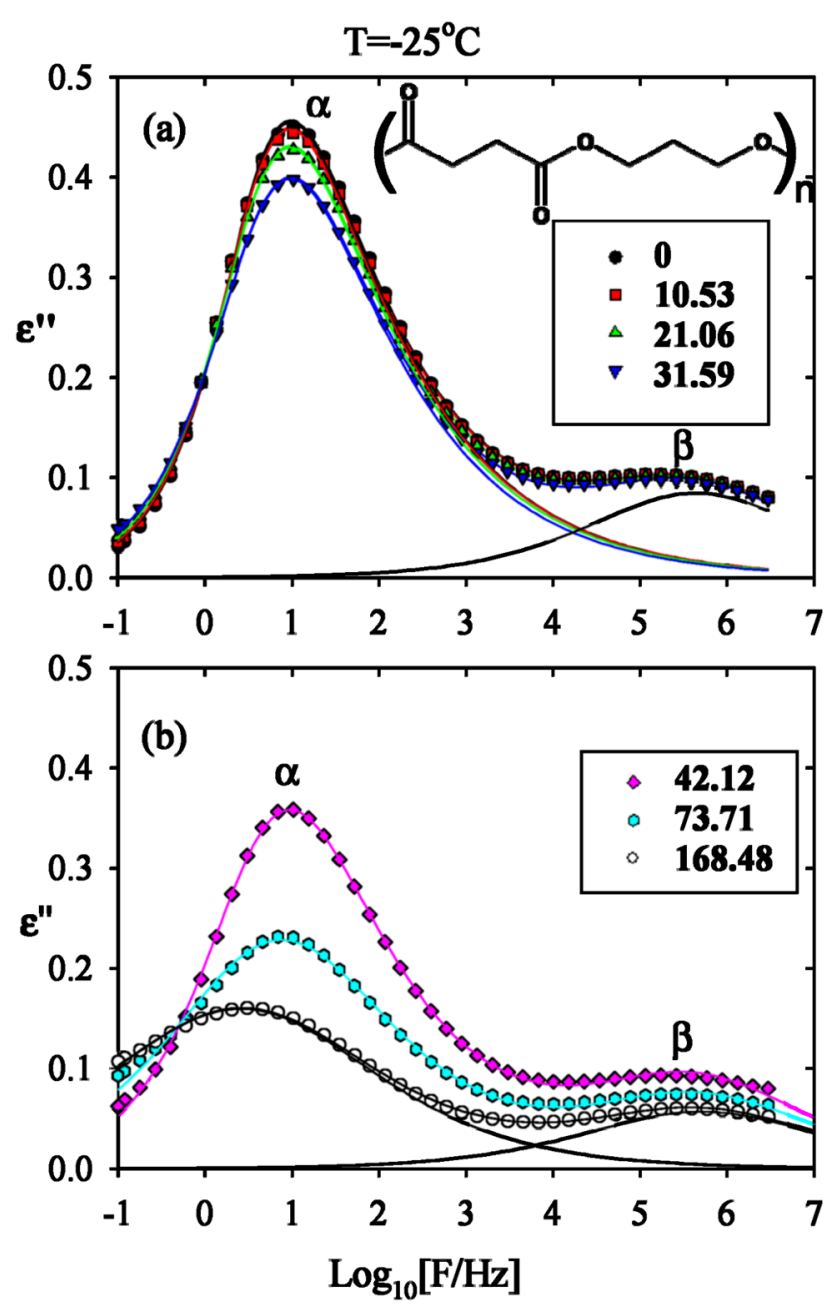

FIG. 1 (color online). Dielectric loss data at $T=-25^{\circ} \mathrm{C}$ as a function of frequency during isothermal crystallization $\left(T_{c}=\right.$ $25^{\circ} \mathrm{C}$ ). Panel (a) shows the induction period where no variation of the $\beta$ relaxation is detected. Panel (b) shows the crystallization period where both $\alpha$ and $\beta$ relaxations decrease significantly as a function of time. The continuous lines are examples of the model Havriliak-Negami function. The inset in (a) is an scheme of poly(propylene succinate). Legends to the symbols indicate the crystallization time in minutes.

accomplished considering both $\alpha$ and $\beta$ relaxations as being Havriliak-Negami (HN) processes [12]. Accordingly, dielectric loss data can be described as $\varepsilon^{\prime \prime}=$ $\operatorname{Im}\left[\varepsilon^{*}\right]=\operatorname{Im}\left\{\varepsilon_{\infty}+\sum_{x=\alpha, \beta} \Delta \varepsilon_{x}\left[1+\left(i \omega \tau_{\mathrm{HN}^{x}}\right)^{b_{x}}\right]^{-c_{x}}\right\}$, where the relaxation strength $\Delta \varepsilon=\varepsilon_{0}-\varepsilon_{\infty}$, where $\varepsilon_{0}$ and $\varepsilon_{\infty}$ are the relaxed and unrelaxed limits of the dielectric constant [12,14], respectively, $\tau_{\mathrm{HN}}$ is the central relaxation time of the relaxation time distribution function, and $b, c$ are the shape parameters which describe, respectively, the symmetric and asymmetric broadening of the relaxation time distribution function [12]. The subscript makes reference to either the $\alpha$ or the $\beta$ relaxation. Additionally, the frequency of maximum loss, $F_{\max }$, can be calculated by means of the following relation: $\frac{1}{2 \pi F_{\max }}=$
$\tau_{\mathrm{HN}}\left[\sin \frac{b \pi}{2+2 c}\right]^{-1 / b}\left[\sin \frac{b c \pi}{2+2 c}\right]^{1 / b}$. The continuous lines in Figs. 1(a) and 1(b) represent the fits of the experimental data to the HN equation with the corresponding deconvolution of the two relaxations. The $\beta$ relaxation can be treated as a symmetric process, $c=1$, during the whole crystallization process. During the first regime [Fig. 1(a)], the $\alpha$ relaxation suffers a significant modification while the $\beta$ relaxation can be well described by the initial parameters corresponding to the initial amorphous sample. In the second regime [Fig. 1(b)], both $\alpha$ and $\beta$ require a modification of the parameters in order to be described by the HN equation. Figure 2 shows the evolution with time of all the HN parameters describing the $\alpha$ and $\beta$ relaxations. The general evolution of the shape parameters of the $\alpha$ relaxation follows what is expected during a crystallization process [2,16]: (i) a reduction of the $\Delta \varepsilon_{\alpha}$ values associated with the reduction of the amorphous mobile phase which is transferred to the crystalline phase, (ii) a reduction of the $b_{\alpha}$ parameter indicating an increase of the broadening, (iii) an increase of $c_{\alpha}$ indicating a symmetrization of the relaxation, and (iv) a reduction of the frequency of maximum loss, $F_{\max }$, associated with the slowing down of the amorphous phase due to the confinement induced by the crystalline phase. The $\beta$ relaxation qualitatively follows the same trend with the exception of the $F_{\max }$ values, which remain essentially unchanged during the crystallization process. This is somehow expected because, due to the local character of the $\beta$ relaxation $[12,14]$, its relaxation time is essentially not affected by the crystalline environment. However, the most streaking fact is the existence of a first regime, denoted in Fig. 2 by a dotted line, where the $\Delta \varepsilon_{\beta}$ remains unchanged while a significant decrease of $\Delta \varepsilon_{\alpha}$ relaxation is observed. Experiments performed in poly(ethylene terephthalate) (PET) have shown that $\Delta \varepsilon_{\beta}$ decreases linearly with the amount of crystalline phase [14]. In order to prove that this relationship also applies for PPS we have accomplished an estimation of the crystallinity by WAXS. Figure 3 shows $\Delta \varepsilon_{\beta}$ as a function of crystallinity. Inserted in Fig. 3 are the diffractograms of the initial sample and the one after the isothermal crystallization experiment. The crystallinity has been estimated as the ratio between the area below the crystalline peaks (continuous lines) and the total area [17]. These results support the use of $1-\Delta \varepsilon_{\beta}(t) / \Delta \varepsilon_{\beta}(0)$ as an estimation of the crystallinity. Accordingly, the first regime observed in Fig. 2 can be identified with the induction period of crystallization. The observed decrease of $\Delta \varepsilon_{\alpha}$ in the induction period indicates a modification of the dynamics of the molten state with respect to the initial one. In order to discuss this time dependence, one has to consider the Frölich-Kirwood equation [18,19], which describes the so-called reduced dielectric strength $f\left(\varepsilon_{0}\right)=$ $\frac{\left(\varepsilon_{0}-\varepsilon_{\infty}\right)\left(2 \varepsilon_{0}+\varepsilon_{\infty}\right)}{\varepsilon_{0}\left(\varepsilon_{\infty}+2\right)^{2}}=\frac{4 \pi \rho N_{a}}{9 k T M} g \mu^{2}$, where $g$ is the correlation factor, $\rho$ is the density, $\mu$ is the dipole moment of the relaxing unit, $M$ is the molecular weight of the repeating unit, $N_{a}$ is Avogadro's number, and $k$ is the Boltzmann constant. The 

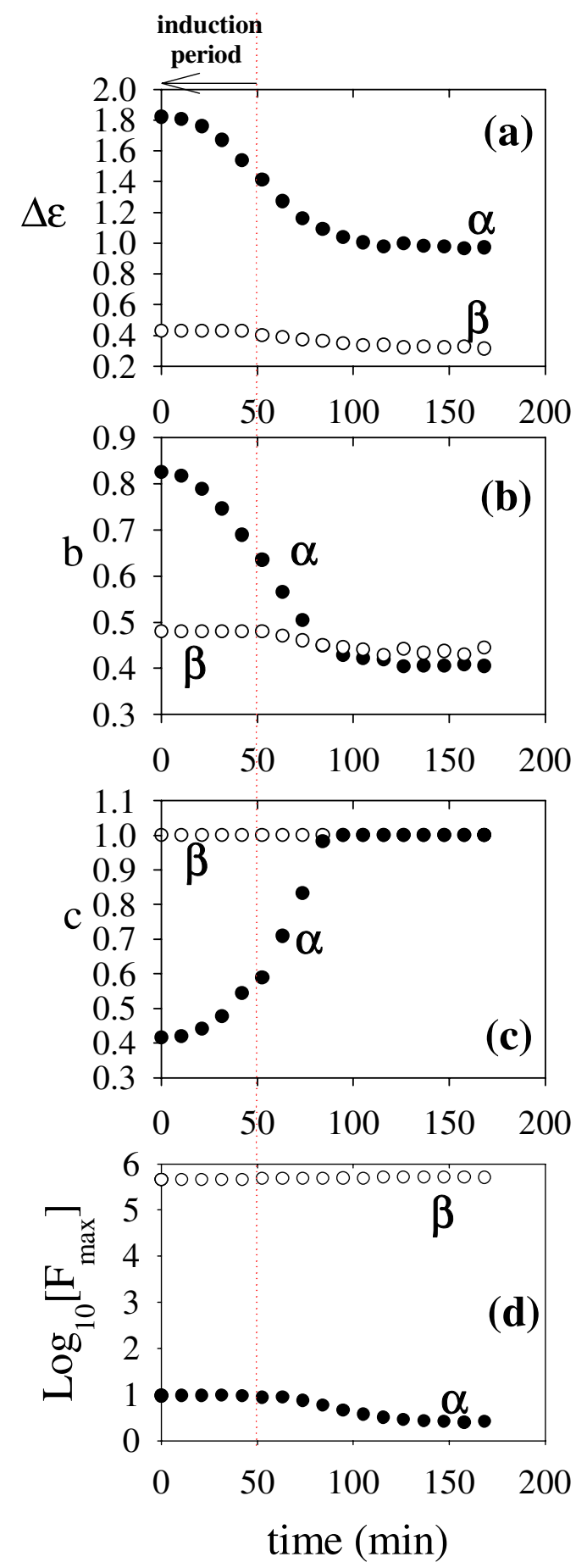

FIG. 2 (color online). Results of the fittings of the dielectric data for the $\alpha(O)$ and $\beta(\bigcirc)$ relaxations to the HavriliakNegami equation. From the top to the bottom: (a) dielectric strength $(\Delta \varepsilon)$; (b) broadening parameter; (c) asymmetry parameter; (d) frequency of maximum loss. All parameters represented as a function of time. Vertical dotted line indicates the limit of the induction period.

values for $\varepsilon_{0}$ and $\varepsilon_{\infty}$ can be obtained from the measured real part of $\varepsilon^{*}$. Figure 4 shows normalized values of $\Delta \varepsilon_{\beta}$, $\Delta \varepsilon_{\alpha}$, and $f\left(\varepsilon_{0}\right)$ during the induction period. The net decrease exhibited by $f\left(\varepsilon_{0}\right)$ while $\Delta \varepsilon_{\beta}$ remains constant can be attributed to a decrease of the correlation factor due to an increment of the intramolecular correlation in the induction period. There are several possibilities to account for the observed increase in the intramolecular correlation in the induction period. Either the formation of fringed micelle nuclei as expected when a spinodal liquid-liquid phase separation is present $[7,20]$ or the formation of mobile "baby nuclei" predicted by computer simulation $[6,21]$ may act as physical cross-links somehow pinning most of the amorphous phase and therefore enhancing the segmental correlation. It is worth mentioning that in experiments performed in PET [14] with different densities induced by thermal treatment, $\Delta \varepsilon_{\alpha}$, show a pronounced decrease without significant changes in $\Delta \varepsilon_{\beta}$. Although this effect was not discussed at that time, it may be an indication of some dynamical changes occurring in the amorphous phase before crystallization.

As far as the shape of the relaxation is concerned, Schönhals and Schlosser have proposed a phenomenological model in which the shape parameters, which characterize the dielectric $\alpha$ relaxation in the frequency domain, can be related to the molecular dynamics at the glass transition $[22,23]$. In this model, the $b$ and $c$ HN parameters are related to two new so-called scaling parameters of the $\alpha$ relaxation $m$ and $n$ by $m=b$ and $n=b c$. This model relates $m$ to large-scale motions while $n$ is controlled by small-scale motions. In our case, the variation of the $m$ and $n$ parameters in the induction period (included in Fig. 4) can be interpreted as a restriction to the large-scale dynamics and an enhancement of the small-scale dynamics. Regardless of the model invoked, our results performed by

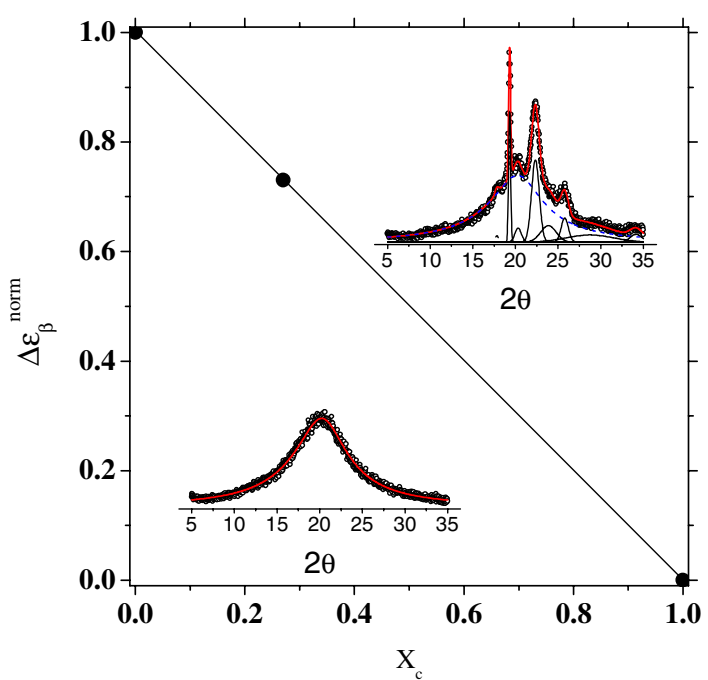

FIG. 3 (color online). Normalized $\Delta \varepsilon_{\beta}$ as a function of WAXS crystallinity. Inserted in the figure are the diffractograms of the initial sample and the one after the isothermal crystallization experiment. The open circles are the experimental points (assuming [14] $\Delta \varepsilon_{\beta}=0$ for $X_{c}=1$ ), the thick continuous lines correspond to the overall fittings, the thin continuous lines are the deconvoluted crystalline peaks, and the dashed line is the amorphous halo. 


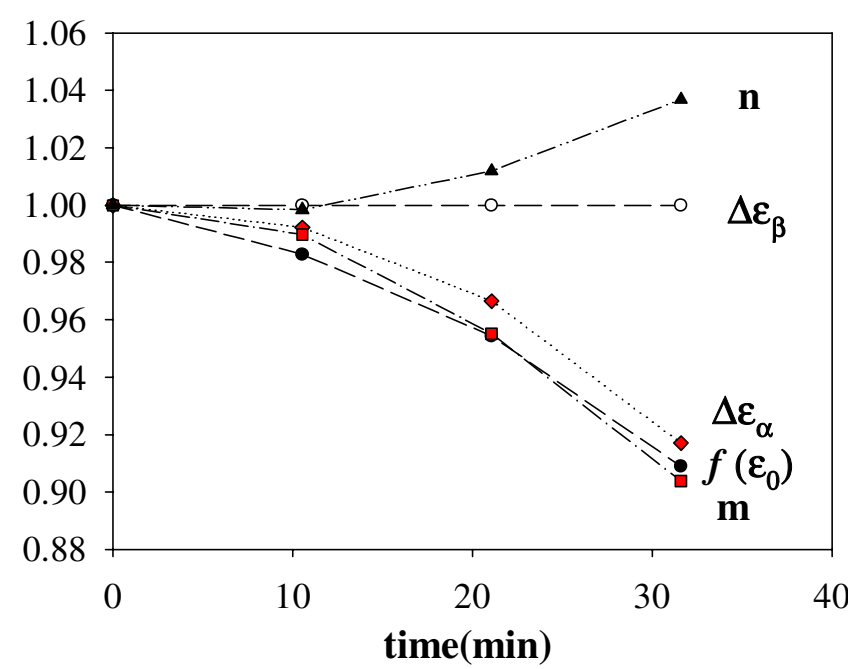

FIG. 4 (color online). Summary of data in the induction period. Dielectric strength $(\Delta \varepsilon)$ for the $\alpha$ (solid diamonds) and $\beta$ (open circles) relaxation. Reduced dielectric strength $f\left(\varepsilon_{o}\right)$ (solid circles). Parameter $m$ (solid squares) and $n$ (solid triangles) from the model of Schönhals and Schlosser [22,23].

a single relaxation technique and capable to detect simultaneously the existence of the crystalline phase, by measuring the $\beta$ relaxation, and the modification of the polymer segmental dynamics, by measuring the $\alpha$ relaxation, clearly support the existence of precursors of crystallization in the induction period.

The authors acknowledge financial support from the MCYT (Grant No. MAT2005-01768), Spain.

[1] M. Imai, K. Kaji, T. Kanaya, and Y. Sakai, Phys. Rev. B 52, 12696 (1995).

[2] K. Fukao and Y. Miyamoto, Phys. Rev. Lett. 79, 4613 (1997).

[3] E. L. Heeley, A. V. Maidens, P. D. Olmsted, W. Bras, I. P. Dolbnya, J.P. A. Faircloug, N. J. Terrill, and A. J. Ryan, Macromolecules 36, 3656 (2003).
[4] G. Strobl, Prog. Polym. Sci. 31, 398 (2006).

[5] P. Olmsted, W. Poon, T. McLeish, T. Terril, and A. J. Ryan, Phys. Rev. Lett. 81, 373 (1998).

[6] P. Welch and M. Muthukumar, Phys. Rev. Lett. 87, 218302 (2001).

[7] R. H. Gee, N. Lacevic, and L. E. Fried, Nat. Mater. 5, 39 (2006).

[8] T. A. Ezquerra, E. Lopez-Cabarcos, B. S. Hsiao, and F. J. Balta-Calleja, Phys. Rev. E 54, 989 (1996).

[9] Z. Zhi-Gang, B.S. Hsiao, E. B. Sirota, P. Agarwal, and S. Srinivas, Macromolecules 33, 978 (2000).

[10] P. Panine, V. Urban, P. Bösecke, and T. Narayan, J. Appl. Crystallogr. 36, 991 (2003).

[11] H. Meyer and F. Müller-Plathe, Macromolecules 35, 1241 (2002).

[12] F. Kremer and A. Schönhals, Broadband Dielectric Spectroscopy (Springer, Berlin, 2002).

[13] A. Sanz, M. Jiménez-Ruiz, A. Nogales, D. Martín y Marero, and T. A. Ezquerra, Phys. Rev. Lett. 93, 015503 (2004).

[14] J. C. Coburn and R.H. Boyd, Macromolecules 19, 2238 (1986).

[15] M. Soccio, L. Finelli, N. Lotti, M. Gazzano, and A. Munari, J. Polym. Sci., B Polym. Phys. (to be published).

[16] T. A. Ezquerra, J. Majszczyk, F. J. Baltà-Calleja, E. LópezCabarcos, K. H. Gardner, and B. S. Hsiao, Phys. Rev. B 50, 6023 (1994).

[17] A. Nogales, T. A. Ezquerra, J. M. García, and F. J. BaltàCalleja, J. Polym. Sci., B Polym. Phys. 37, 37 (1999).

[18] E. Riande and R. Dïaz-Calleja, Electrical Properties of Polymers (Marcel Dekker, New York, 2004).

[19] V. Y. Kramarenko, T. A. Ezquerra, I. Sics, F. J. BaltáCalleja, and V.P. Privalko, J. Chem. Phys. 113, 447 (2000).

[20] K. Kaji, K. Nishida, G. Matsuba, T. Kanaya, and M. Imai, J. Macromol. Sci., Phys. 42, 709 (2003).

[21] M. Muthukumar and P. Welch, Polymer 41, 8833 (2000).

[22] A. Schönhals and E. Schlosser, Colloid Polym. Sci. 267, 125 (1989).

[23] A. Schönhals, F. Kremer, and E. Schlosser, Phys. Rev. Lett. 67, 999 (1991). 